\title{
Small zeros of quadratic forms over algebraic function fields
}

\author{
by \\ Albrecht Pfister (Mainz) \\ Dedicated to J. W. S. Cassels \\ on his 75th birthday
}

1. Introduction. About 40 years ago J. W. S. Cassels [1] proved the following theorem:

Let $q(x)=\sum_{1 \leq i \leq j \leq n} q_{i j} x_{i} x_{j}$ be a quadratic form with integer coefficients $q_{i j} \in \mathbb{Z}$. Assume that $q$ is isotropic over $\mathbb{Q}$. Then there is an $0 \neq a=\left(a_{1}, \ldots, a_{n}\right) \in \mathbb{Z}^{n}$ with $q(a)=0$ such that

$$
|a|=\max \left|a_{i}\right| \leq c_{n} Q^{(n-1) / 2}
$$

where $c_{n}$ is a constant which depends only on $n$ and where $Q=\sum_{i, j}\left|q_{i j}\right|$.

In the addendum to [1] he showed that the exponent $(n-1) / 2$ is best possible by giving an example which was found by M. Kneser. In his book $[3,6.8]$ he gave a better proof and estimate, in particular one can take $c_{n}=3^{(n-1) / 2}$.

For a generalization to totally isotropic subspaces of higher dimension see $[8]$.

Several years later Cassels [2] published his theorem on the representation of a polynomial $f(x)$ in one variable as a sum of $n$ squares: If such a representation is possible over the rational function field $k(x)$ then it is already possible over the polynomial ring $k[x]$. He remarked that the underlying geometrical idea for the proof was essentially the same as in his first paper [1]. This idea is as follows:

Given $a \neq 0$ with $q(a)=0$, intersect the "quadric" $q=0$ with a "line" $l=0$ passing through $a$. If $l$ is chosen carefully then the second intersection point of $q$ and $l$ may be "smaller" than the original point $a$. For the choice of $l$ one has to use the fact that $\mathbb{Z}$ resp. $k[x]$ are euclidean domains.

These results have been generalized in the following directions: 
(1) In 1965 I generalized Cassels' representation theorem (= Darstellungssatz) to an arbitrary quadratic form $q$ over $k$ instead of the "unit form" $q=\langle 1, \ldots, 1\rangle$. See $[4$, Ch. 1] for a proof which includes the case char $k=2$.

(2) In 1975 Raghavan [6] generalized Cassels' zero theorem (= Nullstellensatz) to the ring of integers in an algebraic number field $K$. His estimate for $|a|$ is of the same shape as in (0) but the constant $c_{n}$ now depends on $n=\operatorname{dim} q$ and the degree and the discriminant of $K$. Except for the precise value of the constant $c_{n}$ this paper essentially finishes the number theoretic case.

(3) In 1987 Prestel [5] stated and proved the zero theorem for a rational function field $k(x)$. It reads as follows: There exists $0 \neq a=\left(a_{1}, \ldots, a_{n}\right)$ with $a_{i} \in k[x]$ and $q(a)=0$ such that

$$
\operatorname{deg} a \leq \frac{n-1}{2} \operatorname{deg} Q
$$

where $\operatorname{deg} a=\max _{i}\left(\operatorname{deg} a_{i}\right), \operatorname{deg} Q=\max _{i, j}\left(\operatorname{deg} q_{i j}\right)$.

This may be considered as an additive version of (0) with $c_{n}=1$ for all $n$. The strengthening of the estimate is due to the fact that the valuation on $k(x)$ which is induced by the degree is non-archimedean (= ultrametric).

In the same paper Prestel constructs an example of (a sequence of) isotropic quadratic forms over $\mathbb{R}(x, y)$ in $n=4$ variables with coefficients $q_{i j}$ of degree 2 such that the minimal degree of a non-trivial solution $a$ is unbounded. This proves that one cannot expect results about "small" zeros for function fields in more than one variable.

The aim of the present paper is to prove the Nullstellensatz (Theorem 1) and the Darstellungssatz (Theorem 4) in the remaining open case where $K$ is an algebraic function field in one variable over an arbitrary field $k$. The main difficulty is to find an argument which replaces the euclidean algorithm for $k[x]$. In my proofs this will be the theorem of Riemann-Roch.

I found the main breakthrough three years ago when I spent my sabbatical in Cambridge and enjoyed the privilege of being a Visiting Fellow Commoner of Trinity College.

I have been informed that Dorothea Diers (Münster) has obtained very similar results in her thesis under W. Scharlau but I have not seen any details of her work.

Notation. $k$ is an arbitrary field, $K / k$ is an algebraic function field in one variable. As usual we assume that $K / k$ is finitely generated and that $k$ is algebraically closed in $K$. Divisors of $K / k$ are denoted by latin capitals $A, B, \ldots$, prime divisors by $P$. $\Omega$ denotes the set of all places $P$ of $K / k$, $S=\left\{P_{1}, \ldots, P_{s}\right\}$ is an arbitrary non-empty finite subset of $\Omega$. We write $A=\sum_{P \in \Omega} v_{P}(A) P$. For $P \in \Omega$ the valuation ring of $P$, residue field of $P$ 
and residue degree of $P$ are denoted by $R_{P}, k_{P}$ and $f_{P}$ respectively. $v_{P}$ : $K \rightarrow \mathbb{Z} \cup \infty$ denotes the normalized discrete valuation of $K / k$ corresponding to $P, \pi=\pi_{P}$ is a prime element for $P$. It is well known that $R:=R(S):=$ $\bigcap_{P \notin S} R_{P}$ is a Dedekind ring in $K$ with quot $(R)=K$. For abbreviation we write $R_{\sigma}, k_{\sigma}, f_{\sigma}$ etc. instead of $R_{P_{\sigma}}, k_{P_{\sigma}}, f_{P_{\sigma}}(\sigma=1, \ldots, s)$. Finally, let $f=f(S)=\max \left\{f_{1}, \ldots, f_{s}\right\}$ and let $g$ be the genus of $K / k$.

We consider quadratic forms

$$
q=q(x)=\sum_{\substack{i, j=1 \\ i \leq j}}^{n} q_{i j} x_{i} x_{j} \in K\left[x_{1}, \ldots, x_{n}\right] .
$$

Since char $k=2$ is not excluded $q$ is not supposed to be in diagonal form nor to be non-degenerate. The only standard assumptions about $q$ are:

$$
q \neq 0, \quad \operatorname{dim} q=n \geq 1 .
$$

In Section 2, $q$ is an isotropic form over $K$. This implies $n \geq 2$ since $q=q_{11} x_{1}^{2}$ with $q_{11} \neq 0$ cannot be isotropic. The symmetric bilinear form corresponding to $q$ is given by

$$
q(x, y):=q(x+y)-q(x)-q(y)=\sum_{i \leq j} q_{i j}\left(x_{i} y_{j}+x_{j} y_{i}\right) .
$$

We look for isotropic vectors $0 \neq a=\left(a_{1}, \ldots, a_{n}\right) \in R^{n}$ of $q$. The pole divisor $A$ of $a$ is the smallest non-negative divisor $A \geq 0$ such that $\left(a_{i}\right)+A \geq 0$ for $i=1, \ldots, n$. For $a_{i} \in R$ we see that $A$ is a linear combination of the prime divisors $P_{1}, \ldots, P_{s} \in S$ with coefficients from $\mathbb{N}_{0}: A=\sum_{\sigma=1}^{s} v_{\sigma}(A) P_{\sigma}$. Similarly $Q$ denotes the pole divisor of $q$, i.e. the smallest non-negative divisor such that

$$
\left(q_{i j}\right)+Q \geq 0 \quad(1 \leq i \leq j \leq n) .
$$

$S$ and $q$ are called compatible if all $q_{i j} \in R=R(S)$.

In Section 3 we use a slightly different notation. Here $q$ is a form over $k$ such that the extended form $q \otimes K$ represents a given element $t \in K^{*}$ (over $K)$. We then work with the $(n+1)$-dimensional isotropic form $q \otimes K \perp\langle-t\rangle$ over $K$.

2. The Nullstellensatz. With the terminology introduced in Section 1 let $q$ be an isotropic quadratic form over $K$.

Definition. A vector $a=\left(a_{1}, \ldots, a_{n}\right) \in R^{n}$ with pole divisor $A$ is called a minimal vector of $q$ over $R$ if

(i) $0 \neq a \in R^{n}$,

(ii) $q(a)=0$,

(iii) $\operatorname{deg} A \in \mathbb{N}_{0}$ is minimal (under conditions (i), (ii)). 
Clearly every isotropic form $q$ has at least one minimal vector $a$ over $R=R(S)$ and then $\operatorname{deg} A$ depends only on $K / k, q$ and $S$ but not on $a$.

The first main result of this article is the following:

TheOREM 1 (Nullstellensatz). For every isotropic quadratic form

$$
0 \neq q=q(x)=\sum_{1 \leq i \leq j \leq n} q_{i j} x_{i} x_{j} \in R\left[x_{1}, \ldots, x_{n}\right]
$$

and every minimal vector $0 \neq a=\left(a_{1}, \ldots, a_{n}\right) \in R^{n}$ of $q$ with pole divisor A we have

$$
\operatorname{deg} A \leq n(f+g-1)+\frac{n-1}{2} \operatorname{deg} Q .
$$

Proof. 1 . First we consider the case $n=2$. Here we have

$$
q(x)=q_{11} x_{1}^{2}+q_{12} x_{1} x_{2}+q_{22} x_{2}^{2} .
$$

Without loss of generality we assume $q_{11} q_{22} \neq 0$. [Otherwise $(1,0)$ or $(0,1)$ is an isotropic vector with pole divisor 0.] Then

$$
q(x)=q_{11}\left(x_{1}-c x_{2}\right)\left(x_{1}-c^{\prime} x_{2}\right)
$$

with $c, c^{\prime} \in K$ since $q$ is isotropic. We have

$$
q_{11} c^{2}+q_{12} c+q_{22}=0, \quad c+c^{\prime}=-q_{12} / q_{11}, \quad c c^{\prime}=q_{22} / q_{11} .
$$

By the definition of $Q$ we have

$$
\left(q_{i j}\right)=Q_{i j}-Q
$$

with non-negative divisors $Q_{i j} \geq 0$ (provided $\left.q_{i j} \neq 0\right)(1 \leq i \leq j \leq n)$. This implies $v_{P}\left(q_{22}\right)-v_{P}\left(q_{11}\right)=v_{P}\left(Q_{22}\right)-v_{P}\left(Q_{11}\right)$ for every prime divisor $P$. Consider the principal divisor

$$
\left(c^{2}\right)=2(c)=2 \sum_{P} v_{P}(c) P
$$

and define $\gamma_{P} \in \mathbb{Z}$ by

$$
2 v_{P}(c)=v_{P}\left(Q_{22}\right)-v_{P}\left(Q_{11}\right)+\gamma_{P} .
$$

Then $\left(c c^{\prime}\right)^{2}=\left(q_{22} / q_{11}\right)^{2}$ implies

$$
2 v_{P}\left(c^{\prime}\right)=v_{P}\left(Q_{22}\right)-v_{P}\left(Q_{11}\right)-\gamma_{P}
$$

for all $P$.

Let $C, C^{\prime}$ be the pole divisors of $c, c^{\prime}$. We have

$$
\begin{aligned}
v_{P}(C)+v_{P}\left(C^{\prime}\right)= & \frac{1}{2} \max \left\{v_{P}\left(Q_{11}\right)-v_{P}\left(Q_{22}\right)-\gamma_{P}, 0\right\} \\
& +\frac{1}{2} \max \left\{v_{P}\left(Q_{11}\right)-v_{P}\left(Q_{22}\right)+\gamma_{P}, 0\right\} .
\end{aligned}
$$

We have to distinguish three cases for $P$ :

(i) $v_{P}\left(Q_{11}\right)-v_{P}\left(Q_{22}\right)+\left|\gamma_{P}\right| \leq 0$. Then $v_{P}(C)=v_{P}\left(C^{\prime}\right)=0$. 
(ii) $v_{P}\left(Q_{11}\right)-v_{P}\left(Q_{22}\right)-\left|\gamma_{P}\right| \leq 0<v_{P}\left(Q_{11}\right)-v_{P}\left(Q_{22}\right)+\left|\gamma_{P}\right|$. Then $v_{P}(c) \neq v_{P}\left(c^{\prime}\right)$, hence

$$
\begin{aligned}
\frac{1}{2}\left(v_{P}\left(Q_{22}\right)-v_{P}\left(Q_{11}\right)-\left|\gamma_{P}\right|\right) & =\min \left\{v_{P}(c), v_{P}\left(c^{\prime}\right)\right\}=v_{P}\left(c+c^{\prime}\right) \\
& =v_{P}\left(q_{12}\right)-v_{P}\left(q_{11}\right)=v_{P}\left(Q_{12}\right)-v_{P}\left(Q_{11}\right)
\end{aligned}
$$

and

$$
v_{P}(C)+v_{P}\left(C^{\prime}\right)=v_{P}\left(Q_{11}\right)-v_{P}\left(Q_{12}\right)>0 .
$$

[Note that this case can only occur if $q_{12} \neq 0$.]

(iii) $0<v_{P}\left(Q_{11}\right)-v_{P}\left(Q_{22}\right)-\left|\gamma_{P}\right|$. Then

$$
\begin{aligned}
v_{P}(C)+v_{P}\left(C^{\prime}\right)= & \frac{1}{2}\left(v_{P}\left(Q_{11}\right)-v_{P}\left(Q_{22}\right)-\gamma_{P}\right) \\
& +\frac{1}{2}\left(v_{P}\left(Q_{11}\right)-v_{P}\left(Q_{22}\right)+\gamma_{P}\right) \\
= & v_{P}\left(Q_{11}\right)-v_{P}\left(Q_{22}\right)>0 .
\end{aligned}
$$

With an obvious notation this implies

$$
\begin{aligned}
\operatorname{deg} C+\operatorname{deg} C^{\prime}= & \sum_{2} f_{P}\left(v_{P}\left(Q_{11}\right)-v_{P}\left(Q_{12}\right)\right) \\
& +\sum_{3} f_{P}\left(v_{P}\left(Q_{11}\right)-v_{P}\left(Q_{22}\right)\right) \\
\leq & \sum_{2+3} f_{P} v_{P}\left(Q_{11}\right) \leq \operatorname{deg} Q_{11}=\operatorname{deg} Q .
\end{aligned}
$$

Hence we may assume without loss of generality that $\operatorname{deg} C \leq \frac{1}{2} \operatorname{deg} Q$.

It is now easy to find a "small" isotropic vector $a=\left(a_{1}, a_{2}\right) \in R^{2}$ by solving the linear equation $a_{1}=c a_{2}$ with $a_{1}, a_{2} \in R \backslash\{0\},\left(a_{i}\right)+A \geq 0$, $A \geq 0, \operatorname{deg} A$ minimal. We need $\left(a_{2}\right)+A-C \geq 0$. Then $\left(a_{1}\right)+A=$ $(c)+\left(a_{2}\right)+A=(c)+C+\left(a_{2}\right)+A-C \geq 0$.

For any divisor $D$ let $L(D)=\{d \in K:(d)+D \geq 0\}$. By the theorem of Riemann-Roch this is a finite-dimensional $k$-vector space of dimension

$$
l(D)=\operatorname{deg} D+1-g+i(D)
$$

where $i(D) \geq 0$ is the index of speciality of $D$.

We want: $\operatorname{supp} A \subset S, l(A-C) \geq 1$. Then there is some $0 \neq a_{2} \in$ $L(A-C)$ with $a_{2} \in R, a_{1}=c a_{2} \in R$. It is sufficient to find $A \geq 0$ with $\operatorname{supp} A \subset S$ and $\operatorname{deg}(A-C) \geq g$. Since all multiples of $f$ occur as degrees of divisors which are supported by $S$ we can find such an $A$ with

$$
g \leq \operatorname{deg}(A-C) \leq g+f-1 .
$$

Then $\operatorname{deg} A \leq f+g-1+\operatorname{deg} C \leq f+g-1+\frac{1}{2} \operatorname{deg} Q$.

Note. In the case $n=2$ we have shown that

$$
\operatorname{deg} A \leq f+g-1+\frac{1}{2} \operatorname{deg} Q .
$$


This estimate is better than the estimate of the theorem unless $f=1$ and $g=0$. Another easy estimate is obtained by taking $a_{2}=q_{11}$ and $a_{1}=c a_{2}$. Then

$$
\begin{gathered}
a_{1}^{2}+q_{12} a_{1}+q_{11} q_{22}=0, \\
v_{P}\left(a_{1}\right) \geq \min \left\{v_{P}\left(q_{11}\right), v_{P}\left(q_{12}\right), v_{P}\left(q_{22}\right)\right\} \geq-v_{P}(Q)
\end{gathered}
$$

for all $P \in \Omega$, hence

$$
\left(a_{1}\right)+Q \geq 0, \quad\left(a_{2}\right)+Q \geq 0, \quad A \leq Q, \quad \operatorname{deg} A \leq \operatorname{deg} Q .
$$

This estimate is better than the above estimate if $\frac{1}{2} \operatorname{deg} Q<f+g-1$.

2 . From now on we assume $n \geq 3$. Furthermore, we can assume that either $s=1$ or that $S$ is the exact set of poles of $q$, i.e. $v_{P}(Q)>0$ for all $P \in S$. To see this let $S^{\prime}=\left\{P \in S: v_{P}(Q)>0\right\} \subset S$. Then $f^{\prime}=\max \left\{f_{P}:\right.$ $\left.P \in S^{\prime}\right\} \leq f$. If $\left|S^{\prime}\right| \geq 1$ then application of Theorem 1 for $S^{\prime}$ instead of $S$ yields an isotropic vector $0 \neq a=\left(a_{1}, \ldots, a_{n}\right) \in R\left(S^{\prime}\right)^{n}$ with

$$
\operatorname{deg} A \leq n\left(f^{\prime}+g-1\right)+\frac{n-1}{2} \operatorname{deg} Q \leq n(f+g-1)+\frac{n-1}{2} \operatorname{deg} Q .
$$

If $S^{\prime}=\emptyset$, i.e. $Q=0$, i.e. $q_{i j} \in k$ for all $i, j$, then $S$ can be replaced by any one-point subset $S^{\prime \prime} \subset S$.

The proof will be by contradiction. Hence we start with the

Hypothesis. $0 \neq a \in R^{n}$ is a minimal vector of $q$ with

$$
\operatorname{deg} A>n(f+g-1)+\frac{n-1}{2} \operatorname{deg} Q .
$$

Let $A=\sum_{\sigma=1}^{s} v_{\sigma}(A) P_{\sigma}$. Since $\operatorname{deg} A>0$ we can fix a $\sigma \in\{1, \ldots, s\}$ with $v_{\sigma}(A)>0$. Then $B:=A-P_{\sigma} \geq 0$ and $B<A, \operatorname{deg} B=\operatorname{deg} A-f_{\sigma} \geq$ $\operatorname{deg} A-f$. By our hypothesis we have

$$
\begin{gathered}
\operatorname{deg} A>3(f+g-1), \quad \operatorname{deg} A \geq 3 f+3 g-2, \\
\operatorname{deg} B \geq 2 f+3 g-2>2 g-2 .
\end{gathered}
$$

Therefore

$$
\operatorname{dim} L(B)=\operatorname{deg} B+1-g \geq 2 f+2 g-1 \geq 1 .
$$

Put

$$
V:=\left\{b=\left(b_{1}, \ldots, b_{n}\right) \in K^{n}:\left(b_{i}\right)+B \geq 0 \text { for } i=1, \ldots, n\right\} .
$$

Then $V$ is a $k$-vector space, $\operatorname{dim} V=n(\operatorname{deg} B+1-g) \geq n>0$. For $0 \neq b \in V$ we clearly have $q(b) \neq 0$ since the pole divisor of $b$ is $\leq B<A$.

3. The main idea of the proof is as follows: Join the point $a \in R^{n}$ on the quadric $q=0$ to the point $b \in R^{n}$ off the quadric $q=0$ by a line $l$ and intersect $l$ with this quadric. This yields a second point of intersection

$$
a^{*}:=q(b) a-q(a, b) b \in R^{n} .
$$


For a "good choice" of $b \in V$ we can show that the pole divisor $A^{*}$ of $a^{*}$ satisfies $\operatorname{deg} A^{*}<\operatorname{deg} A$, which contradicts the minimality of $a$.

Let us check that $a^{*} \neq 0$ and $q\left(a^{*}\right)=0$ for any $0 \neq b \in V$. Since $q(b) \neq 0$ and $q(a)=0$ we know that $a, b$ are linearly independent (over $R$ ), hence $a^{*} \neq 0$. Further,

$$
q\left(a^{*}\right)=q(b)^{2} q(a)+q(a, b)^{2} q(b)-q(b) q(a, b) q(a, b)=0 .
$$

4. In order to compute $v_{\sigma}\left(a_{j}^{*}\right)$ for $\sigma \in\{1, \ldots, s\}$ choose $i(\sigma) \in\{1, \ldots, n\}$ such that

$$
v_{\sigma}\left(a_{j}\right) \geq v_{\sigma}\left(a_{i(\sigma)}\right)=-v_{\sigma}(A), \quad j=1, \ldots, n .
$$

Put

$$
c^{(\sigma)}:=b-\frac{b_{i(\sigma)}}{a_{i(\sigma)}} a \in K^{n} .
$$

Then

$$
\begin{aligned}
a^{*} & =q\left(\frac{b_{i(\sigma)}}{a_{i(\sigma)}} a+c^{(\sigma)}\right) a-q\left(a, c^{(\sigma)}\right)\left(\frac{b_{i(\sigma)}}{a_{i(\sigma)}} a+c^{(\sigma)}\right) \\
& =\frac{b_{i(\sigma)}}{a_{i(\sigma)}} q\left(a, c^{(\sigma)}\right) a+q\left(c^{(\sigma)}\right) a-\frac{b_{i(\sigma)}}{a_{i(\sigma)}} q\left(a, c^{(\sigma)}\right) a-q\left(a, c^{(\sigma)}\right) c^{\sigma} \\
& =q\left(c^{(\sigma)}\right) a-q\left(a, c^{(\sigma)}\right) c^{(\sigma)} .
\end{aligned}
$$

Hence

$$
\begin{aligned}
v_{\sigma}\left(a_{h}^{*}\right) & \geq \min _{i, j} v_{\sigma}\left(q_{i j}\right)+\min _{j} v_{\sigma}\left(a_{j}\right)+2 \min _{j} v_{\sigma}\left(c_{j}^{(\sigma)}\right) \\
& \geq-v_{\sigma}(Q)-v_{\sigma}(A)+2 \min _{j} v_{\sigma}\left(c_{j}^{(\sigma)}\right)
\end{aligned}
$$

for all $h=1, \ldots, n$. We want to make $v_{\sigma}\left(c_{h}^{(\sigma)}\right)$ as large as possible. A priori we have

$$
\begin{aligned}
v_{\sigma}\left(c_{h}^{(\sigma)}\right) & =v_{\sigma}\left(b_{h} a_{i(\sigma)}-b_{i(\sigma)} a_{h}\right)-v_{\sigma}\left(a_{i(\sigma)}\right) \\
& \geq \min _{j} v_{\sigma}\left(b_{j}\right) \geq-v_{\sigma}(B) \quad \text { for all } h .
\end{aligned}
$$

By suitable choice of $b \in V$ we want to arrange that

$$
v_{\sigma}\left(c_{j}^{(\sigma)}\right) \geq-v_{\sigma}(B)+\gamma_{\sigma} \quad \text { for all } j \neq i(\sigma)
$$

where the numbers $\gamma_{\sigma} \in \mathbb{N}_{0}(\sigma=1, \ldots, s)$ are chosen later. [Note that $c_{i(\sigma)}^{(\sigma)}=0, v_{\sigma}\left(c_{i(\sigma)}^{(\sigma)}\right)=\infty$.]

Fix for a moment $\sigma, j \neq i(\sigma)$ and $\pi=\pi_{\sigma}$. In the completion $K_{\sigma} \cong$ $k_{\sigma}((\pi))$ of $K$ with respect to $P_{\sigma}$ the element $c_{j}^{(\sigma)}$ has a Laurent series

$$
c_{j}^{(\sigma)}=\pi^{-v_{\sigma}(B)}\left(c_{0}+c_{1} \pi+\ldots\right) \quad \text { with } c_{0}, c_{1}, \ldots \in k_{\sigma} .
$$


$(*)$ is fulfilled for $c_{j}^{(\sigma)}$ iff $c_{0}=c_{1}=\ldots=c_{\gamma_{\sigma}-1}=0$. Let

$$
W:=\bigoplus_{\sigma=1}^{s} \bigoplus_{j \neq i(\sigma)} W_{\sigma, j} \quad \text { with } W_{\sigma, j}:=k_{\sigma}^{\gamma_{\sigma}} .
$$

Then $W$ is a $k$-vector space of dimension $\operatorname{dim} W=(n-1) \sum_{\sigma=1}^{s} f_{\sigma} \gamma_{\sigma}$. The map

$$
\alpha: V \rightarrow W, \quad b \mapsto \bigoplus_{\sigma} \bigoplus_{j}\left(c_{0}, \ldots, c_{\gamma_{\sigma}-1}\right)_{\sigma, j},
$$

is clearly $k$-linear.

$(*)$ is fulfilled for all $\sigma$ and $j$ iff $0 \neq b \in \operatorname{ker} \alpha$. Therefore we impose the condition $\operatorname{dim} V>\operatorname{dim} W$. This gives some upper bound for $\sum f_{\sigma} \gamma_{\sigma}$.

5 . The above estimate for $v_{\sigma}\left(a_{h}^{*}\right)$ leads to a useful estimate for $v_{\sigma}\left(A^{*}\right)$ only in the case

$$
-v_{\sigma}(Q)-v_{\sigma}(A)+2\left(-v_{\sigma}(B)+\gamma_{\sigma}\right) \leq 0 .
$$

Therefore we impose the following conditions on the numbers $\gamma_{\sigma} \in \mathbb{N}_{0}$ :

(1) $\gamma_{\sigma} \leq \gamma_{\sigma}^{*}:=v_{\sigma}(B)+\left[\frac{v_{\sigma}(Q)+v_{\sigma}(A)}{2}\right]$ for each $\sigma=1, \ldots, s$,

(2) $\sum_{\sigma=1}^{s} f_{\sigma} \gamma_{\sigma}<\frac{n}{n-1}(\operatorname{deg} B+1-g)$, i.e. $\operatorname{dim} W<\operatorname{dim} V$.

We compute $\sum f_{\sigma} \gamma_{\sigma}^{*}$ :

(i) For $s \geq 2$ we have $v_{\sigma}(Q) \geq 1$ for all $\sigma$ by our a priori assumption. This gives

$$
\begin{aligned}
\gamma_{\sigma}^{*} & \geq v_{\sigma}(B)+\frac{1}{2} v_{\sigma}(A) \\
\sum f_{\sigma} \gamma_{\sigma}^{*} & \geq \operatorname{deg} B+\frac{1}{2} \operatorname{deg} A \geq \operatorname{deg} B+\frac{1}{2}(\operatorname{deg} B+1) \\
& =\frac{3}{2}(\operatorname{deg} B+1)-1 \geq \frac{n}{n-1}(\operatorname{deg} B+1-g)-1 \\
& =\frac{1}{n-1} \operatorname{dim} V-1
\end{aligned}
$$

since $n \geq 3$.

(ii) For $s=1$ we have $A=m P$ with $m>0, B=(m-1) P$. Then

$$
\begin{aligned}
\gamma_{1}^{*} & \geq m-1+\left[\frac{m}{2}\right] \geq m-1+\frac{m-1}{2}=\frac{3}{2}(m-1), \\
f_{1} \gamma_{1}^{*} & =f \gamma_{1}^{*} \geq \frac{3}{2} f(m-1)=\frac{3}{2} \operatorname{deg} B .
\end{aligned}
$$

Hence again (for $n \geq 3$ )

$\frac{1}{n-1} \operatorname{dim} V-1 \leq \frac{1}{2} \operatorname{dim} V-1=\frac{1}{2}(\operatorname{deg} B+1-g)-1 \leq \frac{3}{2} \operatorname{deg} B \leq f_{1} \gamma_{1}^{*}$ since $-\frac{1}{2}(1+g) \leq 0 \leq \operatorname{deg} B$. 
Since $\sum f_{\sigma} \gamma_{\sigma}^{*} \in \mathbb{N}_{0}$ this shows that $\sum f_{\sigma} \gamma_{\sigma}^{*}$ is greater than or equal to the largest integer below $\operatorname{dim} V /(n-1)$ in both cases.

Let now $\left(\gamma_{1}, \ldots, \gamma_{s}\right)$ be any $s$-tuple with $0 \leq \gamma_{\sigma} \leq \gamma_{\sigma}^{*}$ and $\sum_{\sigma=1}^{s} f_{\sigma} \gamma_{\sigma}$ $>0$. Replacing a fixed $\gamma_{\tau}>0$ by $\gamma_{\tau}-1$ reduces this sum to $\sum f_{\sigma} \gamma_{\sigma}-f_{\tau}$ where $1 \leq f_{\tau} \leq f$. This shows that for any closed interval $I \subset\left[0, \frac{1}{n-1} \operatorname{dim} V\right)$ of length $\geq f$ there exists a system $\left(\gamma_{1}, \ldots, \gamma_{s}\right) \in \mathbb{N}_{0}^{s}$ with $\gamma_{\sigma} \leq \gamma_{\sigma}^{*}$ for all $\sigma$ and $\sum f_{\sigma} \gamma_{\sigma} \in I$.

6 . We are now able to derive an estimate for $\operatorname{deg} A^{*}$. From our Hypothesis we have

say

$$
\begin{aligned}
& \operatorname{deg} B \geq \operatorname{deg} A-f>n(g-1)+(n-1) f+\frac{n-1}{2} \operatorname{deg} Q, \\
& \frac{1}{2} \operatorname{deg} Q+f<\frac{1}{n-1}(\operatorname{deg} B+n(1-g)), \\
& \frac{1}{2} \operatorname{deg} Q+\operatorname{deg} B+f<\frac{n}{n-1}(\operatorname{deg} B+1-g)=\frac{1}{n-1} \operatorname{dim} V,
\end{aligned}
$$

$$
\frac{1}{2} \operatorname{deg} Q+\operatorname{deg} B+f+\varepsilon=\frac{1}{n-1} \operatorname{dim} V \quad \text { with } \varepsilon>0 .
$$

Choose $I=\left[\frac{1}{2} \operatorname{deg} Q+\operatorname{deg} B+\varepsilon / 2, \frac{1}{2} \operatorname{deg} Q+\operatorname{deg} B+f+\varepsilon / 2\right]$. Then we find a system $\left(\gamma_{1}, \ldots, \gamma_{s}\right)$ as above such that $\sum f_{\sigma} \gamma_{\sigma} \in I$, i.e.

$$
\frac{1}{2} \operatorname{deg} Q+\operatorname{deg} B<\sum f_{\sigma} \gamma_{\sigma}<\frac{1}{n-1} \operatorname{dim} V .
$$

Put $C:=\sum_{\sigma=1}^{s} \gamma_{\sigma} P_{\sigma}$. Let $W$ be the $k$-vector space corresponding to $\left(\gamma_{1}, \ldots, \gamma_{s}\right)$. Then $\operatorname{dim} W=(n-1) \sum f_{\sigma} \gamma_{\sigma}<\operatorname{dim} V$.

Choose

$$
0 \neq b \in \operatorname{ker}\{\alpha: V \rightarrow W\}, \quad a^{*}=q(b) a-q(a, b) b \in R^{n}
$$

and let $A^{*}$ denote the pole divisor of $a^{*}$. The estimates of part 4 imply:

$$
\begin{aligned}
v_{\sigma}\left(a_{h}^{*}\right) & \geq-v_{\sigma}(Q)-v_{\sigma}(A)+2 \min _{j} v_{\sigma}\left(c_{j}^{(\sigma)}\right) \\
& \geq-v_{\sigma}(Q)-v_{\sigma}(A)-2 v_{\sigma}(B)+2 \gamma_{\sigma}, \\
v_{\sigma}\left(A^{*}\right) & \leq v_{\sigma}(Q)+v_{\sigma}(A)+2 v_{\sigma}(B)-2 \gamma_{\sigma} \in \mathbb{N}_{0}, \\
\operatorname{deg} A^{*} & =\sum_{\sigma=1}^{s} f_{\sigma} v_{\sigma}\left(A^{*}\right) \leq \operatorname{deg} Q+\operatorname{deg} A+2 \operatorname{deg} B-2 \operatorname{deg} C<\operatorname{deg} A,
\end{aligned}
$$

since $\operatorname{deg} Q+2 \operatorname{deg} B-2 \operatorname{deg} C<0$ by (**). This contradicts the minimality of the isotropic vector $a$ and proves the theorem.

From Theorem 1 we can easily derive the following more general but slightly weaker 
TheOREM 2. Let $q \neq 0$ be an isotropic quadratic form over $K$ of dimension $n \geq 2$. Let $Q$ be the pole divisor of $q$. For $Q \neq 0$ let $S$ be the exact support of $Q$, and $f=f(S)$. For $Q=0$ choose $S=\{P\}$ and $f=f(S)=f_{P}$ with an arbitrary prime divisor $P \in \Omega$, e.g. such that $f_{P}=f_{0}:=\min \left\{f_{P}: P \in \Omega\right\}$. In addition, let $D$ be any divisor of $K$ such that

$$
\operatorname{deg} D \geq n(f+g-1)+\frac{n-1}{2} \operatorname{deg} Q+g .
$$

Then there exists a non-trivial vector $b=\left(b_{1}, \ldots, b_{n}\right)$ with $q(b)=0$ and $\left(b_{i}\right)+D \geq 0(i=1, \ldots, n)$.

Proof. Define $R=R(S)$ and choose a minimal vector $a \in R^{n}$ of $q$ with pole divisor $A \geq 0$. By Theorem 1 we have

$$
\operatorname{deg} A \leq n(f+g-1)+\frac{n-1}{2} \operatorname{deg} Q .
$$

We put $b=t \cdot a$ with $t \in K^{*}$ and try to choose $t$ such that $\left(b_{i}\right)+D \geq 0$ $(i=1, \ldots, n)$. Clearly $q(b)=0$. Since $\left(a_{i}\right)+A \geq 0, b_{i}=t a_{i}$ we need

$$
\left(a_{i}\right)+(t)+D \geq 0 .
$$

This is true if $(t)+D \geq A$, i.e. $t \in L(D-A)$. So we need $\operatorname{dim} L(D-A)>0$. This is certainly the case if

$$
\operatorname{deg} D-\operatorname{deg} A+1-g>0, \quad \operatorname{deg} D \geq \operatorname{deg} A+g .
$$

By our assumption on $D$ this inequality is true.

Note. If we write $D=D_{1}-D_{2}$ with (disjoint) non-negative divisors $D_{1}, D_{2}$ then $\left(b_{i}\right)+D_{1} \geq 0$. This means $b_{i} \in R_{1}:=R\left(S_{1}\right)$ where $S_{1}=$ $\operatorname{supp}\left(D_{1}\right), 0 \neq b \in R_{1}^{n}, q(b)=0$. Here $S_{1}$ and $q$ are not compatible in general.

Instead of scaling the vector $a$ we can also scale the quadratic form $q$ without changing the equation $q(a)=0$. Thereby we can derive

THEOREM 1'. Every isotropic form $q$ admits an isotropic vector $a \neq 0$ such that

$$
\operatorname{deg} A \leq \frac{3 n-1}{2}\left(f_{0}+g-1\right)+\frac{n-1}{2} \operatorname{deg} Q
$$

where $f_{0}=\min \left\{f_{P}: P \in \Omega\right\}$.

Proof. Let $P_{0} \in \Omega$ be such that $f_{P_{0}}=f_{0}$. We try to find $q_{0} \in K^{*}$ such that $\left(q_{0} q_{i j}\right)+m P_{0} \geq 0$ for suitable $m \in \mathbb{N}_{0}$. We need $\left(q_{0}\right)+m P_{0}-Q \geq 0$. For this it is enough that

$$
g \leq m f_{0}-\operatorname{deg} Q \leq g+f_{0}-1, \quad \text { or } \quad m=\left[\frac{\operatorname{deg} Q+f_{0}+g-1}{f_{0}}\right] .
$$


We apply Theorem 1 to the isotropic form $q^{\prime}=q_{0} q$ with pole divisor $Q^{\prime} \leq m P_{0}$. This leads to an isotropic vector $a \in R_{0}^{n}$ (where $R_{0}=R\left(\left\{P_{0}\right\}\right)$ ) such that

$$
\begin{aligned}
\operatorname{deg} A & \leq n\left(f_{0}+g-1\right)+\frac{n-1}{2} \operatorname{deg} Q^{\prime} \leq n\left(f_{0}+g-1\right)+\frac{n-1}{2} m f_{0} \\
& \leq \frac{3 n-1}{2}\left(f_{0}+g-1\right)+\frac{n-1}{2} \operatorname{deg} Q .
\end{aligned}
$$

Note. Depending on the special values of $f_{0}, f, g$ and $n$, Theorem $1^{\prime}$ or Theorem 1 may give a "smaller" isotropic vector $a$ for $q$.

EXAMPLE 1. For the rational function field $K=k(x)$ and the set $S=$ $\{\infty\}$ with $v_{\infty}(u)=-\operatorname{deg} u$ for all $u \in K$ we have $R=k[x], f=1, g=0$. If then $q_{i j} \in R(1 \leq i \leq j \leq n)$, Theorem 1 coincides with the theorem of Prestel [5]. The estimate is then best possible for all $n$.

EXAMPLE 2. Let char $k \neq 2$ and let $q=\left\langle 1, q_{2}, q_{3}\right\rangle$ be an anisotropic ternary quadratic form over $k$. Consider for $g \in \mathbb{N}_{0}$ the function field

$$
K=k(t, u) \quad \text { with }-u^{2}=q_{2} t^{2 g+2}+q_{3} .
$$

It has genus $g$. All prime divisors $P$ of $K$ have even degree, since otherwise $q \otimes k_{P}$ would be isotropic over the odd-degree extension $k_{P} / k$, which contradicts Springer's theorem. There is one place $P=\infty$ over the infinite place of $k(t)$, it has $k_{\infty}=k\left(\sqrt{-q_{2}}\right), f_{\infty}=2$. For $S=\{\infty\}$ we get $R=R(S)=k[t, u]$ and $Q=0$ since $q$ is a "constant" form. The vector $a=\left(u, t^{g+1}, 1\right)$ is a minimal vector of $q$ over $R$ with $\operatorname{deg} A=2(g+1)$. The minimality follows since $u$ must occur in at least one component of every isotropic vector $a \in R^{3}$ and since $u$ has pole divisor $U=(g+1) \infty$ with $\operatorname{deg} U=2(g+1)$. The estimate of Theorem 1 gives the weaker estimate

$$
\operatorname{deg} A \leq n(f+g-1)=3(g+1) .
$$

[For $g=0$ Theorem 1 is essentially sharp since $\operatorname{deg} A \leq 3$ automatically implies $\operatorname{deg} A \leq 2$.]

ExAmple 3 . Let $k=\mathbb{Q}_{\text {pyth }}$ be the pythagorean closure of $\mathbb{Q}$. Then $k$ is real with Pythagoras number $p(k)=1$. It is known (see e.g. [4, Ch. 7]) that $p(k(t)) \geq 3$ and that there exists a polynomial $h=h(t)$ of degree 4 which is a sum of 3 but not of 2 squares in $k[t]$. Put $K=k(t, u)$ with $u^{2}=-h$. Then $K$ is non-real. In particular, all prime divisors of $K / k$ have even degree, the place $\infty$ has degree $f_{\infty}=2, t$ has pole divisor $\infty, u$ has pole divisor $2 \infty$. Let $S=\{\infty\}$ and $R=[t, u]$ as above. Then $f=2$ and $g=1$ (since $h$ has degree 4$)$.

We take the constant ternary quadratic form $q=\langle 1,1,1\rangle$ with pole divisor $Q=0$. From the equation $u^{2}+h=0$ it follows that the form $\langle 1\rangle \oplus q=4 \times\langle 1\rangle$ is isotropic over $K$. As is well known this implies that 
$q \otimes K$ is isotropic as well. Let $a=\left(a_{1}, a_{2}, a_{3}\right)$ be a minimal vector of $q$ over $R$. Then $a_{i}=b_{i}+c_{i} u(i=1,2,3)$ with $b_{i}, c_{i} \in k[t]$, and $a_{i}$ has pole divisor $m_{i} \cdot \infty$ with

$$
m_{i}=\max \left\{\operatorname{deg} b_{i}, \operatorname{deg} c_{i}+2\right\}
$$

where deg is the ordinary degree on $k[t]$. Theorem 1 gives the estimate $\operatorname{deg} A \leq 3(f+g-1)=6$ for the pole divisor $A$ of $a$. We want to show that this estimate is sharp (recall that $\operatorname{deg} A$ is even):

Assume $\operatorname{deg} A \leq 4$. Then $c_{1}, c_{2}, c_{3}$ must be constants from $k$ (not all zero). The equation

$$
0=\sum_{i=1}^{3}\left(b_{i}+c_{i} u\right)^{2}=\sum b_{i}^{2}-h \sum c_{i}^{2}+2 u \sum b_{i} c_{i}
$$

implies:

1) $\sum b_{i} c_{i}=0$, i.e. $b_{1}, b_{2}, b_{3}$ are linearly dependent over $k$, say $b_{3}=$ $\lambda b_{1}+\mu b_{2}$ with $\lambda, \mu \in k$,

2) $\sum_{i=1}^{3} b_{i}^{2}=h \sum_{i=1}^{3} c_{i}^{2}=h \cdot c^{2}$ with $0 \neq c \in k$.

By normalizing we can arrange $c=1$. Then

$$
h=b_{1}^{2}+b_{2}^{2}+\left(\lambda b_{1}+\mu b_{2}\right)^{2} \quad \text { in } k[t] .
$$

Put

$$
\tau:=\sqrt{\lambda^{2}+\mu^{2}+1} \in k, \quad \nu=\frac{\lambda^{2} \mu+\tau}{\lambda^{2}+1}, \quad \omega=\frac{\lambda(\mu-\tau)}{\lambda^{2}+1} .
$$

An easy computation yields

$$
h=\left(b_{1}+\omega b_{2}\right)^{2}+\left(\lambda b_{1}+\nu b_{2}\right)^{2},
$$

i.e. $h$ is a sum of 2 squares in $k[t]$ : Contradiction.

Final remarks to Theorems $1,1^{\prime}, 2$

1. Since the estimates for $\operatorname{deg} A$ grow with the number $n=\operatorname{dim} q$ they can only be sharp if all proper subforms of $q$ are anisotropic over $K$. For certain fields this ensures an a priori upper bound for $n$. If e.g. $K$ is a $C_{i}$-field then every quadratic form of dimension $>2^{i}$ over $K$ is isotropic, which means that we can suppose $n \leq 2^{i}+1$.

2. If $g \neq 1$ then there is a well-known upper bound for the greatest common divisor $\delta$ of all possible divisor degrees ( $\delta$ is called the index of $K / k)$ and for the minimal prime divisor degree $f_{0}$, namely $\delta \leq f_{0} \leq|2 g-2|$. Then $f_{0}$ or $f=f(S)$ could be eliminated from the estimates in many cases.

3. If $g=1$ then $f_{0}=\delta$ and $\delta$ can be any natural number. For concrete examples where $k$ is a $p$-adic field see e.g. [7, Cor. 13a].

3. The Darstellungssatz. Let now $q \neq 0$ be a quadratic form over $k$ and suppose that $q \otimes K$ represents a given element $t \in K^{*}$. We look for a 
representation

$$
q(u)=q\left(u_{1}, \ldots, u_{n}\right)=t
$$

for which the pole divisor $U$ of $u$ has small degree. The result will be slightly different depending on whether $q \otimes K$ is isotropic or not. For the anisotropic case we first need a variant of Theorem 1 . Here a non-trivial solution in $K$ of the equation

$$
q\left(a_{1}, \ldots, a_{n}\right)-t a_{0}^{2}=0
$$

automatically satisfies $a_{0} \neq 0, q\left(a_{1}, \ldots, a_{n}\right) \neq 0$, and leads to a solution of (1) by putting $u_{i}=a_{i} / a_{0}$. Since then $a_{0}$ is (up to sign) completely determined by $q, t$ and the vector $a=\left(a_{1}, \ldots, a_{n}\right)$ we measure the "smallness" of a solution of (2) be the degree of the pole divisor $A$ of $a$ (as in Section 2).

Let $N \geq 0$ be the divisor of zeros of $t$, let $T \geq 0$ be the pole divisor of $t$, i.e. $(t)=N-T$. We have unique decompositions $N=2 N_{0}+N_{1}, T=2 T_{0}+T_{1}$ with $v_{P}\left(N_{1}\right), v_{P}\left(T_{1}\right) \in\{0,1\}$ for all prime divisors $P$. Since $\operatorname{deg} N=\operatorname{deg} T$ we have

$$
\begin{aligned}
2 \operatorname{deg}\left(T_{0}+T_{1}-N_{0}\right) & =\operatorname{deg} T+\operatorname{deg} T_{1}-2 \operatorname{deg} N_{0} \\
& =\operatorname{deg} T_{1}+\operatorname{deg} N-2 \operatorname{deg} N_{0} \\
& =\operatorname{deg} T_{1}+\operatorname{deg} N_{1} \geq 0 .
\end{aligned}
$$

If $\operatorname{supp} T \neq \emptyset$ let $S=\operatorname{supp} T$, otherwise let $S=\{P\}$ be any one-point set from $\Omega$. Put $R=R(S)$ and $f=f(S)$. Call a non-trivial solution $\left(a_{1}, \ldots, a_{n}, a_{0}\right)$ of $(2)$ resp. its vector $a=\left(a_{1}, \ldots, a_{n}\right)$ minimal if $\operatorname{deg} A$ is as small as possible.

THEOREM 3. Under the above assumptions there exists a non-trivial solution of (2) with $a \in R^{n}$. If a is minimal then

$$
\operatorname{deg} A \leq(n+1)(f+g-1)+\operatorname{deg}\left(T_{0}+T_{1}-N_{0}\right) .
$$

Proof. The first statement is clear since $q \otimes K$ represents $t$ and $K=$ quot $(R)$. Suppose now that $a$ is minimal but $\operatorname{deg} A$ does not satisfy the estimate of Theorem 3. Then $A=\sum_{\sigma=1}^{s} v_{\sigma}(A) P_{\sigma}$ with $P_{\sigma} \in S, s=|S|$, and $v_{\sigma_{0}}(A)>0$ for at least one $\sigma_{0}$ since $\operatorname{deg} A>0$ by our assumption. Fix $\sigma_{0}$ and put $B:=A-P_{\sigma_{0}}$. Then $0 \leq B<A$ and $\operatorname{deg} B=\operatorname{deg} A-f_{0} \geq \operatorname{deg} A-f$. As in the proof of Theorem 1 we shall construct a vector $0 \neq a^{*} \in R^{n}$ and an element $a_{0}^{*} \neq 0$ such that $q\left(a^{*}\right)=t a_{0}^{* 2}$ but $\operatorname{deg} A^{*}<\operatorname{deg} A$. This contradicts the minimality of $a$ and proves the theorem.

From (2) we have

$$
\left(a_{i}\right)+A \geq 0 \quad \text { for } i=1, \ldots, n,
$$

hence 


$$
\begin{aligned}
(t)+2\left(a_{0}\right)+2 A & \geq 0, \\
N_{1}+2 N_{0}-2 T_{0}+2\left(a_{0}\right)+2 A & \geq 0, \\
2\left(N_{0}-T_{0}+\left(a_{0}\right)+A\right) & \geq 0,
\end{aligned}
$$

and finally,

$$
\left(a_{0}\right)+A+N_{0}-T_{0} \geq 0 .
$$

We look for vectors $b=\left(b_{1}, \ldots, b_{n}\right) \in K^{n}$ and elements $b_{0} \in K$ such that

$$
\begin{gathered}
\left(b_{i}\right)+B \geq 0 \quad \text { for } i=1, \ldots, n, \\
\left(b_{0}\right)+B+N_{0}-T_{0}-T_{1} \geq 0 .
\end{gathered}
$$

The $k$-vector space $V$ of all $(n+1)$-tuples $\left(b_{1}, \ldots, b_{n}, b_{0}\right)$ satisfying (4) has dimension

$$
\operatorname{dim} V \geq(n+1)(\operatorname{deg} B+1-g)+\operatorname{deg}\left(N_{0}-T_{0}-T_{1}\right) .
$$

From (2) and (4) we conclude

$$
\left(t a_{0}^{2}\right)+2 A \geq 0, \quad\left(t b_{0}^{2}\right)+T-N+2 B+2\left(N_{0}-T_{0}-T_{1}\right) \geq 0,
$$

i.e. $\left(t b_{0}^{2}\right)+2 B-T_{1}-N_{1} \geq 0$, hence

$$
\left(t b_{0}^{2}\right)+2 B \geq 0 \text { and }\left(t a_{0} b_{0}\right)+A+B \geq 0 .
$$

For all $i=0, \ldots, n$ define

$$
\begin{aligned}
& a_{i}^{*}:=\left(q(b)-t b_{0}^{2}\right) a_{i}-\left(q(a, b)-2 t a_{0} b_{0}\right) b_{i}, \\
& a^{*}:=\left(a_{1}^{*}, \ldots, a_{n}^{*}\right), \quad A^{*}:=\text { pole divisor of } a^{*} .
\end{aligned}
$$

It follows that

$$
\begin{gathered}
q\left(a^{*}\right)=t a_{0}^{* 2}, \\
\left(a_{i}^{*}\right)+A+2 B \geq 0 \quad \text { for } i=1, \ldots, n, \\
\left(a_{0}^{*}\right)+A+2 B+N_{0}-T_{0} \geq 0 .
\end{gathered}
$$

In particular, we have $a^{*} \in R^{n}$.

Since $B<A$ we cannot have $q(b)-t b_{0}^{2}=0$ unless $b=0$ and $b_{0}=0$. If $0 \neq\left(b_{1}, \ldots, b_{n}, b_{0}\right) \in V$ this implies that $\left(b, b_{0}\right)$ is independent of $\left(a, a_{0}\right)$ over $K$, hence $a^{*} \neq 0$. We have to choose $\left(b, b_{0}\right)$ in such a way that $\operatorname{deg} A^{*}<$ $\operatorname{deg} A$. As in the proof of Theorem 1 we choose for each $\sigma \in\{1, \ldots, s\}$ an index $i(\sigma) \in\{1, \ldots, n\}$ such that $v_{\sigma}(A)=-v_{\sigma}\left(a_{i(\sigma)}\right)$ and put

$$
c_{j}^{(\sigma)}:=b_{j}-\frac{b_{i(\sigma)}}{a_{i(\sigma)}} a_{j} \quad(j=0, \ldots, n) .
$$

Then we get

$$
a_{j}^{*}=\left(q\left(c^{(\sigma)}\right)-t c_{0}^{(\sigma) 2}\right) a_{j}-\left(q\left(a, c^{(\sigma)}\right)-2 t a_{0} c_{0}^{(\sigma)}\right) c_{j}^{(\sigma)}
$$


with $c_{i(\sigma)}^{(\sigma)}=0$,

$$
\begin{aligned}
& v_{\sigma}\left(c_{j}^{(\sigma)}\right)+v_{\sigma}(B) \geq 0 \quad(j=1, \ldots, n), \\
& v_{\sigma}\left(t_{0} c_{0}^{(\sigma) 2}\right)+2 v_{\sigma}(B) \geq 0 .
\end{aligned}
$$

By a suitable choice of $\left(b, b_{0}\right) \in V$ we want to arrange for stronger inequalities

$$
\begin{aligned}
& v_{\sigma}\left(c_{j}^{(\sigma)}\right)+v_{\sigma}(B)-\gamma_{\sigma} \geq 0 \quad \text { for } i(\sigma) \neq j \in\{1, \ldots, n\}, \\
& v_{\sigma}\left(t c_{0}^{(\sigma) 2}\right)+2 v_{\sigma}(B)-2 \gamma_{\sigma} \geq 0
\end{aligned}
$$

where the $\gamma_{\sigma} \in \mathbb{N}_{0}$ are chosen later. If (9) holds then $a^{*}$ satisfies

$$
v_{\sigma}\left(a_{j}^{*}\right)+v_{\sigma}(A)+2 v_{\sigma}(B)-2 \gamma_{\sigma} \geq 0 \quad(j=1, \ldots, n) .
$$

For fixed $\sigma$ condition (9) puts $n f_{\sigma} \gamma_{\sigma} k$-linear equations on the vector $\left(b, b_{0}\right) \in$ $V$, that is, $n \sum_{\sigma=1}^{s} f_{\sigma} \gamma_{\sigma}$ equations altogether. We take

$$
\gamma_{\sigma}=v_{\sigma}(B)+ \begin{cases}0 & \text { for } \sigma \neq \sigma_{0} \\ 1 & \text { for } \sigma=\sigma_{0}\end{cases}
$$

Then $n \sum f_{\sigma} \gamma_{\sigma}=n\left(\operatorname{deg} B+f_{0}\right)<\operatorname{dim} V$, since

$$
\begin{aligned}
\operatorname{dim} & V-n\left(\operatorname{deg} B+f_{0}\right) \\
& \geq(n+1) \operatorname{deg} B+(n+1)(1-g)+\operatorname{deg}\left(N_{0}-T_{0}-T_{1}\right)-n\left(\operatorname{deg} B+f_{0}\right) \\
& =\operatorname{deg} B-n f_{0}-(n+1)(g-1)-\operatorname{deg}\left(T_{0}+T_{1}-N_{0}\right) \\
& \geq \operatorname{deg} A-(n+1)(f+g-1)-\operatorname{deg}\left(T_{0}+T_{1}-N_{0}\right)>0
\end{aligned}
$$

by our assumption. Therefore there exists $0 \neq\left(b, b_{0}\right) \in V$ with (9). By (10) the pole divisor $A^{*}$ of $a^{*}$ satisfies $v_{\sigma}\left(A^{*}\right) \leq v_{\sigma}(A)$ for $\sigma \neq \sigma_{0}$ and

$$
v_{\sigma_{0}}\left(A^{*}\right) \leq \begin{cases}v_{\sigma_{0}}(A)-2 & \text { for } v_{\sigma_{0}}(A) \geq 2, \\ 0 & \text { for } v_{\sigma_{0}}(A)=1\end{cases}
$$

In any case we get $v_{\sigma_{0}}\left(A^{*}\right)<v_{\sigma_{0}}(A)$, hence $\operatorname{deg} A^{*}<\operatorname{deg} A$ : Contradiction.

Notes. 1. For a prime divisor $P \in \operatorname{supp}\left(N_{1}+T_{1}\right)$ the validity of equation (1), when read over the completion $K_{P}$ of $K$, implies that $q \otimes K_{P}$, hence $q \otimes k_{P}$ must be isotropic. By Springer's theorem this implies that $\operatorname{deg} P=$ $\left[k_{P}: k\right]$ is even.

2. For $t \in k^{*}$, i.e. $T=N=0$, Theorem 3 coincides with Theorem 1 for the constant quadratic form $q \perp\langle-t\rangle$.

ExAmple 4 . Let $K=k(x)$ be the rational function field, and let $t=$ $f(x) \in k[x]$ be a squarefree polynomial. If the anisotropic form $q$ (which remains anisotropic over $K$ ) represents $t$ over $K$ then $\operatorname{deg} f=d=2 m$ is even and the pole divisor $T$ of $t$ is given by $T=2 T_{0}$ with $T_{0}=m \cdot \infty, f=$ 
$f_{\infty}=1, g=0$. Theorem 3 gives us a representation $q\left(a_{1}, \ldots, a_{n}\right)=t a_{0}^{2}$ with

$$
a_{i} \in k[x]=R(\infty), \quad \operatorname{deg} a_{i} \leq 0+\operatorname{deg} T_{0}=m \quad(i=1, \ldots, n) .
$$

Then $0 \neq q(a) \in k[x]$ with $\operatorname{deg} q(a) \leq 2 m$. Since $f$ is squarefree we get $0 \neq a_{0} \in k[x]$ with $\operatorname{deg} a_{0} \leq 0$, i.e. $a_{0} \in k^{*}$. This proves the original representation theorem (for anisotropic form $q$ ).

We are now ready to state and prove the representation theorem for an arbitrary algebraic function field $K / k$ with genus $g$.

THEOREM 4 (Darstellungssatz). Let $q$ be a quadratic form over $k, \operatorname{dim} q=$ $n$. Suppose that $t \in K^{*}$ is represented by the form $q \otimes K$. Let $N=2 N_{0}+N_{1}$, $T=2 T_{0}+T_{1}$ and $S \subset \Omega, f=f(S)$ be as before. Then there exists a vector $u=\left(u_{1}, \ldots, u_{n}\right) \in K^{n}$ with pole divisor $U$ such that $q(u)=t$ and

$$
\operatorname{deg} U \leq(n+1)(f+g-1)+\operatorname{deg}\left(T_{0}+T_{1}\right)+\frac{1}{2} \operatorname{deg} N_{1}
$$

or

$$
\operatorname{deg} U \leq n(f+g-1)+\operatorname{deg} T .
$$

[In the "reduced" case the first (second) estimate holds if $q \otimes K$ is anisotropic (isotropic).]

Proof. Since the estimates for $\operatorname{deg} U$ grow with $n$ we can assume that $t$ is not represented by $\widetilde{q} \otimes K$ for any proper $k$-subform $\widetilde{q}$ of $q$ (otherwise replace $q$ by $\widetilde{q}$ with $\operatorname{dim} \widetilde{q}<\operatorname{dim} q$ ). We then call $q$ reduced with respect to $t$. In particular, $q$ is non-defective, i.e. $q=q^{\prime} \perp q^{\prime \prime}$ with $q^{\prime}=\operatorname{rad} q=\left\langle q_{1}, \ldots, q_{r}\right\rangle$ anisotropic over $k$ and $q^{\prime \prime}$ regular over $k$. (See e.g. [4, Ch. 1]; for char $k \neq 2$ one has $q^{\prime}=0, q$ regular.)

First case: $q \otimes K$ anisotropic. Here we can start with a non-trivial solution of $q\left(a_{1}, \ldots, a_{n}\right)-t a_{0}^{2}=0$ according to Theorem 3 and put $u_{i}=$ $a_{i} / a_{0}(i=1, \ldots, n), u=a / a_{0} \in K^{n}$.

A pole $P$ of $u_{i}$ either comes from a pole of $a_{i}$ or from a zero of $a_{0}$. In the first case we have $P \in S$. Let $U=U_{1}+U_{2}$ with $U_{1} \geq 0, U_{2} \geq 0$, $\operatorname{supp} U_{1} \subset S, S \cap \operatorname{supp} U_{2}=\emptyset$. The equation $q(a)=t a_{0}^{2}$ shows:

1) For $P \notin S$ we have $v_{P}\left(a_{i}\right) \geq 0, v_{P}(q(a)) \geq 0, v_{P}(t) \geq 0$ and $v_{P}(q(a))=v_{P}(t)+2 v_{P}\left(a_{0}\right)$. Hence

$$
v_{P}\left(\frac{a_{i}}{a_{0}}\right)+\frac{1}{2} v_{P}(q(a)) \geq-v_{P}\left(a_{0}\right)+\frac{1}{2} v_{P}(q(a)) \geq 0 .
$$

This implies $v_{P}\left(U_{2}\right) \leq \frac{1}{2} v_{P}(q(a))$ for every $P \notin S$, i.e.

$$
\operatorname{deg} U_{2} \leq \frac{1}{2} \sum_{P \notin S} f_{P} v_{P}(q(a)) \text {. }
$$


2) For $P \in S$ we have $v_{P}\left(a_{i}\right)+v_{P}(A) \geq 0, v_{P}(t)+v_{P}(T) \geq 0$ and again $2 v_{P}\left(a_{0}\right)=v_{P}(q(a))-v_{P}(t)$. Hence

$$
v_{P}\left(\frac{a_{i}}{a_{0}}\right)+v_{P}(A)+\frac{1}{2} v_{P}(q(a))-\frac{1}{2} v_{P}(t) \geq 0
$$

where $v_{P}(A)+\frac{1}{2} v_{P}(q(a)) \geq 0$. This implies $v_{P}\left(U_{1}\right) \leq v_{P}(A)+\frac{1}{2} v_{P}(q(a))+$ $\frac{1}{2} v_{P}(T)$, hence

$$
\operatorname{deg} U_{1} \leq \operatorname{deg} A+\frac{1}{2} \operatorname{deg} T+\frac{1}{2} \sum_{P \in S} f_{P} v_{P}(q(a)) .
$$

Since $\sum_{\text {all } P} f_{P} v_{P}(q(a))=\operatorname{deg}(q(a))=0$ the two estimates imply

$$
\operatorname{deg} U=\operatorname{deg} U_{1}+\operatorname{deg} U_{2} \leq \operatorname{deg} A+\frac{1}{2} \operatorname{deg} T=\operatorname{deg} A+\operatorname{deg} N_{0}+\frac{1}{2} \operatorname{deg} N_{1} .
$$

With the estimate of Theorem 3 for $\operatorname{deg} A$ this gives

$$
\operatorname{deg} U \leq(n+1)(f+g-1)+\operatorname{deg}\left(T_{0}+T_{1}\right)+\frac{1}{2} \operatorname{deg} N_{1} .
$$

Second case: $q \otimes K$ isotropic. Here we first show that $q^{\prime} \otimes K$ must be anisotropic (if $q$ is reduced). Suppose that $q^{\prime} \otimes K$ is isotropic, say $q_{r}=$ $\sum_{i=1}^{r-1} q_{i} c_{i}^{2}$ with $c_{i} \in K$. Let $t=q\left(u_{1}, \ldots, u_{n}\right)=q^{\prime}\left(u_{1}, \ldots, u_{r}\right)+q^{\prime \prime}\left(u_{r+1}, \ldots\right.$ $\left.\ldots, u_{n}\right)$ be any representation with $u_{i} \in K$. Then

$$
q^{\prime}\left(u_{1}, \ldots, u_{r}\right)=\sum_{i=1}^{r} q_{i} u_{i}^{2}=\sum_{i=1}^{r-1} q_{i}\left(u_{i}^{2}+c_{i}^{2} u_{r}^{2}\right)=\sum_{i=1}^{r-1} q_{i}\left(u_{i}+c_{i} u_{r}\right)^{2} .
$$

Hence $t$ is represented by $\widetilde{q} \otimes K$ where $\widetilde{q}=\left\langle q_{1}, \ldots, q_{r-1}\right\rangle \perp q^{\prime \prime}$ is a proper subform of $q$ (over $k$ ): Contradiction.

Let now $0 \neq a \in R(S)^{n}$ be a solution of $q(a)=0$ which satisfies the condition of Theorem 1, i.e. $a$ has pole divisor $A$ with $\operatorname{deg} A \leq n(f+g-1)$. [Note that $q$ is constant, i.e. $Q=0$.] Assume for a moment that $q(a, b)=0$ for all vectors $b \in k^{n}$. By linearity this would imply $q(a, b)=0$ for all $b \in K^{n}$, i.e. $0 \neq a \in \operatorname{rad}(q \otimes K)=q^{\prime} \otimes K$. In other words, $q^{\prime}(a)=0$, which is a contradiction. Hence we can choose a vector $0 \neq b \in k^{n}$ such that $a_{0}:=q(a, b) \in K^{*}$. Clearly $\left(a_{0}\right)+A \geq 0$, in particular $a_{0} \in R=R(S)$.

We can now find a good representation of $t$. Put $u:=b+\lambda a$ with some $\lambda \in K$. Then

$$
q(u)=q(b)+\lambda q(a, b)=q(b)+\lambda a_{0},
$$

i.e.

$$
q(u)=t \Leftrightarrow \lambda=\frac{t-q(b)}{a_{0}} \Leftrightarrow u=b+(t-q(b)) \cdot \frac{a}{a_{0}} .
$$

Let $C$ be the pole divisor of $c:=a / a_{0}$. Since $b \in k^{n}$ and $q(b) \in k$ this shows that the pole divisor $U$ of $u$ satisfies $U \leq T+C$. It remains to estimate 
$\operatorname{deg} C$. For every $P \in \Omega$ we have

$v_{P}\left(a_{0}\right)+v_{P}(A) \geq 0 \quad$ and $\quad v_{P}\left(\frac{a_{i}}{a_{0}}\right)+v_{P}\left(a_{0}\right)+v_{P}(A) \geq 0 \quad(i=1, \ldots, n)$,

hence $v_{P}\left(c_{i}\right)+\left(v_{P}\left(a_{0}\right)+v_{P}(A)\right) \geq 0$ for $c_{i}=a_{i} / a_{0}$, i.e. $v_{P}(C) \leq v_{P}\left(a_{0}\right)+$ $v_{P}(A)$. This gives

$$
\operatorname{deg} C=\sum_{P} f_{P} v_{P}(C) \leq \sum_{P} f_{P} v_{P}\left(a_{0}\right)+\sum_{P} f_{P} v_{P}(A)=\operatorname{deg} A,
$$

since $\sum f_{P} v_{P}\left(a_{0}\right)=\operatorname{deg}\left(a_{0}\right)=0$. Therefore

$\operatorname{deg} U \leq \operatorname{deg} C+\operatorname{deg} T \leq \operatorname{deg} A+\operatorname{deg} T \leq n(f+g-1)+\operatorname{deg} T$.

Note. If $q(u)=t$ then the poles $P \in \operatorname{supp} T$ must turn up with suitable multiplicity among the poles of $u_{i}$ for at least one $i \in\{1, \ldots, n\}$. This shows that $U=T_{0}+T_{1}+U^{\prime}$ with a non-negative divisor $U^{\prime} \geq 0$ of degree $\operatorname{deg} U^{\prime} \leq(n+1)(f+g-1)+\frac{1}{2} \operatorname{deg} N_{1}$ or $\operatorname{deg} U^{\prime} \leq n(f+g-1)+\operatorname{deg} T_{0}$.

It does not seem possible to prescribe the prime divisors $P \in \operatorname{supp} U^{\prime}$ since this would amount to knowing the zeros of $a_{0}$.

\section{References}

[1] J. W. S. Cassels, Bounds for the least solutions of homogeneous quadratic equations, Proc. Cambridge Philos. Soc. 51 (1955), 262-264. Addendum, ibid. 52 (1956), 604.

[2] - On the representation of rational functions as sums of squares, Acta Arith. 9 (1964), 79-82.

[3] -, Rational Quadratic Forms, London Math. Soc. Monographs 13, Academic Press, London, 1978.

[4] A. Pfister, Quadratic Forms with Applications to Algebraic Geometry and Topology, London Math. Soc. Lecture Note Ser. 217, Cambridge Univ. Press, 1995.

[5] A. Prestel, On the size of zeros of quadratic forms over rational function fields, J. Reine Angew. Math. 378 (1987), 101-112.

[6] S. Raghavan, Bounds for minimal solutions of Diophantine equations, Nachr. Akad. Wiss. Göttingen 1975 (9), 109-114.

[7] P. Roquette, Analytic theory of elliptic functions over local fields, Hamburg. Math. Einzelschr. 1, Vandenhoeck \& Ruprecht, Göttingen, 1970.

[8] H. P. Schlickewei and W. M. Schmidt, Quadratic forms which have only large zeros, Monatsh. Math. 105 (1988), 295-311.

Fachbereich Mathematik

Universität Mainz

55099 Mainz, Germany

E-mail: pfister@mat.mathematik.uni-mainz.de 